\title{
Error Analysis of MLFMA With Closed-Form Expressions
}

\author{
Mert Kalfa ${ }^{\circledR}$, Member, IEEE, Vakur B. Ertürk ${ }^{\circledR}$, Senior Member, IEEE, and Özgür Ergül ${ }^{\circledR}$, Senior Member, IEEE
}

\begin{abstract}
The current state-of-the-art error control of the multilevel fast multipole algorithm (MLFMA) is valid for any given error threshold at any frequency, but it requires a multiple-precision arithmetic framework to be implemented. In this work, we use asymptotic approximations and curve-fitting techniques to derive accurate closed-form expressions for the error control of MLFMA that can be implemented in common fixed-precision computers. Moreover, using the proposed closed-form expressions in conjunction with the state-of-the-art scheme, we report novel design curves for MLFMA that can be used to determine achievable error limits, as well as the minimum box sizes that can be solved with a given desired error threshold for a wide range of machine precision levels.
\end{abstract}

Index Terms-Error analysis, low-frequency breakdown, multilevel fast multipole algorithm (MLFMA), multiple-precision arithmetic.

\section{INTRODUCTION}

$\mathbf{T}$ HE multilevel fast multipole algorithm (MLFMA), which is a hierarchical extension of the fast multipole method, is a popular full-wave electromagnetic solver that can be applied to the solution of extremely large problems due to its $O(N \log N)$ complexity for $N$ unknowns. This relatively low complexity is achieved by diagonalizing the translation operator that enables the computation of interactions between basis and testing functions in a group-by-group manner [1], [2]. Due to the limited and fixed machine precision of most computing platforms, the diagonalized form of the translation operator becomes numerically unstable as the truncation number of the infinite summation over spherical Hankel functions increases beyond a certain threshold. This is the well-known low-frequency breakdown problem of MLFMA [3].

In one of our previous publications in the context of multiple-precision arithmetic, we have proposed an error control scheme for MLFMA that is valid at all frequencies, which can also take into account the machine precision of the computing platform, bringing a whole new outlook

Manuscript received July 9, 2020; revised January 16, 2021; accepted February 28, 2021. Date of publication April 6, 2021; date of current version October 6, 2021. (Corresponding author: Mert Kalfa.)

Mert Kalfa is with the Department of Electrical and Electronics Engineering, Bilkent University, 06800 Ankara, Turkey, and also with the Huawei Turkey Research and Development Center, 34768 İstanbul, Turkey (e-mail: kalfa@ee.bilkent.edu.tr).

Vakur B. Ertürk is with the Department of Electrical and Electronics Engineering, Bilkent University, 06800 Ankara, Turkey.

Özgür Ergül is with the Department of Electrical and Electronics Engineering, Middle East Technical University, 06800 Ankara, Turkey.

Color versions of one or more figures in this article are available at https://doi.org/10.1109/TAP.2021.3070086.

Digital Object Identifier 10.1109/TAP.2021.3070086 on MLFMA simulations [4]. Given the box size and the desired relative error threshold, the proposed scheme can provide the optimum truncation numbers and the minimum required machine precision levels at all frequencies for the first time in the literature, whereas the previous methods were limited to only high-frequency problems [5]-[8] or modified implementations [9].

In this work, we address two important shortcomings of the state-of-the-art error control of MLFMA, namely, the difficulty in implementing [4] and lack of intuitive observations and design guidelines for the universal error control of MLFMA. First, the error control scheme we present in [4] requires the computation of the diagonalized Green's function for different orders of spherical harmonics, which may or may not be handled by the native precision of the computing platform. Hence, accurate implementation of [4] for computing the truncation numbers requires a reconfigurable multiple-precision framework, such as the one given in [10]. This presents a challenge as a multiple-precision environment may not be available to the practitioners of MLFMA. Even if a multiple-precision environment is available, the increase in implementation complexity may not be desirable. Second, although the previously reported truncation numbers and machine precision levels in [4] provide accurate and important information about the error control, they may not be intuitive for the practitioners who would design their solvers from the ground up according to [4] but may not want to use a multiple-precision framework. Therefore, we believe that insightful and intuitive design curves for the error control of MLFMA that is valid for a wide range of frequencies are sorely needed.

As a response to the above shortcomings, the contribution of this article is twofold. First, we provide simple closed-form expressions for error control at low frequencies (or small boxes) for the first time in the literature in the form of curve-fit and asymptotic formulas. Together with the widely used excess bandwidth formula (EBF) in [5] that is valid for large boxes, our proposed expressions provide a complete error control scheme that can be implemented in common fixed-precision computers without any need for a multiple-precision implementation. Second, we present novel design curves for the lowest possible errors that can be attained with a given machine precision and the minimum translation distances and box sizes that can be computed with a given error threshold, using our novel closed-form expressions in conjunction with the machine precision estimation techniques in [4]. We believe that the proposed novel closed-form expressions and design curves 
will provide the practitioners of MLFMA with a rigorous and intuitive guideline on how to implement MLFMA on both fixed- and variable-precision platforms, such as CPUs, GPUs, and FPGAs, of the state-of-the-art technology.

The rest of this article is organized as follows. Section II briefly describes our previously reported error control scheme and the commonly used EBF. Section III introduces our proposed expressions for error control at low frequencies. Section IV presents the results of our analyses of achievable error limits and presents design curves for accurate error control of MLFMA. Concluding remarks are provided in Section V. An $e^{-i \omega t}$ time convention, where $\omega=2 \pi f$ and $f$ is the operating frequency, is assumed and suppressed throughout this article.

\section{ERror Control Scheme of MLFMA}

Spherical wave expansion of the free-space Green's function can be derived using Gegenbauer's addition theorem as

$$
\begin{aligned}
& \frac{\exp (i k|\vec{w}+\vec{v}|)}{4 \pi|\vec{w}+\vec{v}|} \\
& \quad=\frac{i k}{4 \pi} \sum_{t=0}^{\infty}(-1)^{t}(2 t+1) j_{t}(k v) h_{t}^{(1)}(k w) P_{t}(\hat{w} \cdot \hat{v})
\end{aligned}
$$

where $P_{t}$ is the Legendre polynomial of order $t ; j_{t}$ and $h_{t}^{(1)}$ are the spherical Bessel and Hankel functions of the first kind, respectively. Note that (1) is only valid for $w=|\vec{w}|>$ $v=|\vec{v}|$, where $\vec{w}$ and $\vec{v}$ represent the translation and shift vectors, respectively. MLFMA uses the diagonal form of the Green's function given in (1) by transforming the spherical waves as integrals over the plane-wave spectrum [1].

Using a truncation number of $\tau$ for the infinite summation in (1), the truncation error can be derived by assuming only the $(\tau+1)$ th term's dominant contribution as

$$
\hat{\epsilon} \approx k R(2 \tau+3)\left|j_{\tau+1}(k v) h_{\tau+1}^{(1)}(k w) P_{\tau+1}(\hat{w} \cdot \hat{v})\right|,
$$

where $R=|\vec{w}+\vec{v}|$. In (2), the maximum error occurs when the translation distance $w$ is the smallest and the total shift distance $v$ is the greatest. Therefore, the maximum error is achieved when a one-box-buffer scheme is used with $w=2 a$, with $a$ representing the box edge length, while $v=\left|\left[\begin{array}{lll}a & a & a\end{array}\right]^{\mathrm{T}}\right|=a \sqrt{3}$, where $\mathrm{T}$ represents the matrix transpose.

Using the large argument approximation of the Bessel and Hankel functions in (2), commonly used EBF [5]-[7] can be derived to determine the truncation number for the onebox-buffer scheme as

$$
\tau \approx k a \sqrt{3}+2.18\left(d_{0}\right)^{2 / 3}(k a)^{1 / 3},
$$

where $d_{0} \triangleq-\log _{10}\left(\epsilon_{d}\right)$ is the desired digits of accuracy with $\epsilon_{d}$ being the desired relative error threshold. Although being a widely adopted method, (3) is only valid for large box sizes or high-frequency problems due to the large argument approximations.

With the recently introduced novel error control scheme [4], the relative error of the free-space Green's function for a given truncation number $\tau$ can be calculated using the large order approximation of Bessel and Hankel functions [11] as

$$
\hat{\boldsymbol{\epsilon}} \approx \frac{R}{\sqrt{w v}}\left|\frac{P_{\tau+1}(\hat{w} \cdot \hat{v}) \psi_{j}\left(0.5 \psi_{h}-i \psi_{h}^{-1}\right)}{\sqrt{\tanh \gamma_{j} \tanh \gamma_{h}}}\right|,
$$

where $\psi_{j}$ and $\psi_{h}$ are defined as

$$
\begin{aligned}
& \psi_{j} \triangleq \exp \left[(\tau+1.5)\left(\tanh \gamma_{j}-\gamma_{j}\right)\right] \\
& \psi_{h} \triangleq \exp \left[(\tau+1.5)\left(\tanh \gamma_{h}-\gamma_{h}\right)\right]
\end{aligned}
$$

with

$$
\begin{aligned}
& \gamma_{j}=\operatorname{sech}^{-1}\left(\frac{k v}{\tau+1.5}\right), \\
& \gamma_{h}=\operatorname{sech}^{-1}\left(\frac{k w}{\tau+1.5}\right) .
\end{aligned}
$$

Unlike EBF, (4) cannot be solved for $\tau$ in closed-form; therefore, the truncation number is found numerically. Once the truncation number is found from (4), the required machine precision to compute the translation operator can be found with the procedure outlined in [4], which is not repeated here for brevity. However, an important assumption of this procedure is worth repeating: The minimum machine precision with the novel scheme is defined so that the machine precision is able to handle each of the individual separate numbers and elementary functions in the translation operator, as well as all of the intermediate combinations (i.e., products, summations, and integrations) before the computation of the final result. Hence, the resulting minimum requirement for machine precision is actually a supremum value of possible minimum precision levels and assumes the worst case in terms of implementation. In practice, the minimum machine precision requirement can be further reduced by carefully tracking the magnitude range of each number and intermediate function of the translation operator for each problem and making sure that the registers do not overflow or underflow during computations.

\section{Closed-Form EXPRESSIONS FOR ERror CONTROL AT LOW FREQUENCIES}

Truncation numbers computed with (4) for a given desired error threshold agree well with EBF for large box sizes and high-frequency problems [4]. However, for small box sizes and low-frequency problems, EBF fails as it relies on the large-argument approximation of Bessel and Hankel functions. On the other hand, the requirement for a multiple-precision framework and a lack of closed-form solution complicates the use of (4) for most implementations. Therefore, in this section, we derive simplified closed-form expressions that do not require a multiple-precision environment for small boxes and low-frequency problems for the first time in the literature. Together with widely used EBF at high frequencies as their counterpart, the novel closed-form expressions allow easy error control of MLFMA for all frequencies and translation distances.

To simplify (2) for low-frequency problems where $k w \ll 1$ and $k v \ll 1$, we use the small-argument approximations of 
the Bessel and Hankel functions [11] as

$$
\begin{aligned}
J_{t}(z) & =\frac{(z / 2)^{t}}{(t+1) !}, \\
H_{t}^{(1)}(z) & =\left(\frac{-i}{\pi}\right) \frac{(t) !}{(z / 2)^{t}} .
\end{aligned}
$$

Then, the conversion from cylindrical to spherical Bessel and Hankel functions is performed using

$$
\begin{aligned}
j_{t}(z) & =\sqrt{\frac{\pi}{2 z}} J_{t+0.5}(z), \\
h_{t}^{(1)}(z) & =\sqrt{\frac{\pi}{2 z}} H_{t+0.5}^{(1)}(z) .
\end{aligned}
$$

For the simplification of the Legendre polynomials, we use Rodrigues' formula [11, (8.6.18)] and integration by parts to calculate the root-mean-square (RMS) value over the range $\hat{w} \cdot \hat{v} \in[-1,1]$ to obtain

$$
\sqrt{\int_{-1}^{1} P_{t}^{2}(z) \mathrm{z}}=\sqrt{\frac{2}{2 t+1}}
$$

Substituting (9)-(13) into (2), we obtain the approximate truncation error for low-frequency problems as

$$
\hat{\epsilon} \approx \frac{R}{\sqrt{w v}}\left(\frac{v}{w}\right)^{\tau+1.5} \sqrt{\frac{2}{2 \tau+3}} .
$$

Note that the closed-form expression in (14) can be used to estimate the truncation error for different translation and shift distances. However, the common approach in practice is to select the highest possible truncation number for the worst case in terms of error, which corresponds to the one-box-buffer scheme. Therefore, we can further simplify (14) by assuming a one-box-buffer scheme and substituting $\vec{w}=\left[\begin{array}{lll}0 & 2 a & 0\end{array}\right]$ and $\vec{v}=\left[\begin{array}{lll}a & a & a\end{array}\right]$, resulting in

$$
\hat{\epsilon} \approx 1.782\left(\frac{\sqrt{3}}{2}\right)^{\tau+1.5} \sqrt{\frac{2}{2 \tau+3}},
$$

where the direction of $\vec{w}$ in a one-box-buffer scheme does not affect the result. The closed-form expression in (15) can be used as a complement to EBF for small boxes to estimate the worst case errors in a one-box-buffer scheme. On the other hand, the closed-form expression in (14) can be used if different truncation numbers are to be used for different translation distances. An important insight for (14) and (15) is that they do not depend on the box sizes. This behavior is consistent with the low-frequency harmonics of Green's function when the multipole expansion is explicitly used [12].

The expressions given in (14) and (15) still have room for improvement. Specifically, they still require numerical implementations to search for truncation numbers for a given desired error threshold and may suffer from inaccuracies due to the RMS approximation of the Legendre polynomials. Therefore, we provide yet another alternative for the low-frequency scenarios in the form of curve-fit functions of (4), which we consider to be the gold standard among all other alternatives presented in this work.

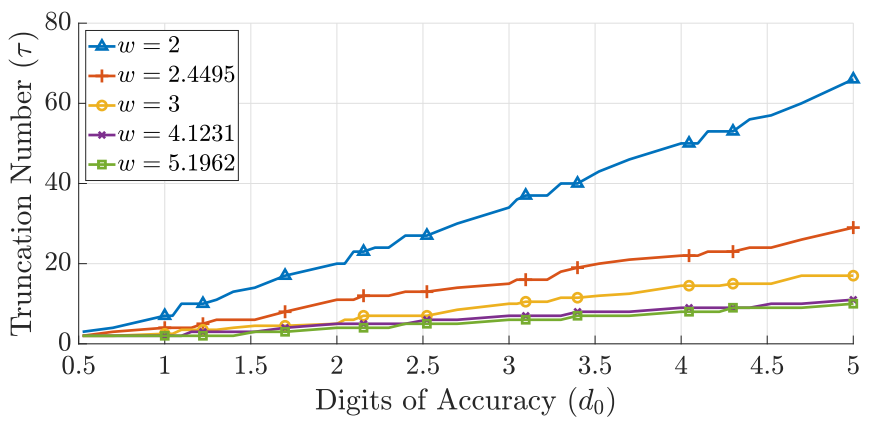

Fig. 1. Truncation numbers obtained via (4) for $a=\lambda / 2048$.

To generate the data for curve-fitting, we have computed truncation numbers using (4) for the following range of parameters.

1) Desired Error Threshold: $\epsilon_{d} \in[3.3 \mathrm{e}-1,1 \mathrm{e}-5]$.

2) Box Sizes: $a \in[\lambda / 2048, \lambda]$.

3) Translation Vectors $\vec{w}$ : All possible vectors within a parent box with symmetries removed (17 unique cases).

4) Shift Vectors $\vec{v}$ : From corners, edge centers, and face centers of the source box to those of the observation box (62 unique cases).

After the required truncation numbers for the above scenarios are computed, we assume that the truncation error only depends on the translation distance normalized to the box size, i.e., $w / a$, consistent with our findings of (14) and (15). As an example, computed truncation numbers for a box size of $\lambda / 2048$ for some $w / a$ values are illustrated in Fig. 1. A clear observation from Fig. 1 is that the relation between $d_{0}$ and $\tau$ can be closely represented by an affine transformation, where the slope and offset parameters of the transformation being functions of $w / a$ are

$$
\tau \approx f_{1}(w / a) d_{0}+f_{2}(w / a) .
$$

Then, the slope, $f_{1}(\cdot)$, and the offset, $f_{2}(\cdot)$, of the affine transformation that depend on $w / a$ are approximated by double-exponential functions that are empirically found to be best fit as

$$
\begin{aligned}
& f_{1}(x)=3.073 \mathrm{e} 4 \times e^{-4.064 x}+10.34 \times e^{-0.3562 x}, \\
& f_{2}(x)=-28.63 \times e^{-1.071 x}-1.168 \mathrm{e} 8 \times e^{-8.620 x} .
\end{aligned}
$$

Note that the closed-form expressions given in (16)-(18) can be used as an alternative to (14) if different truncation numbers are to be used for different translation distances within the same parent box of MLFMA. Similar to the derivation of (15), we can simplify (16) for the maximum error of the onebox-buffer scheme with $w=2 a$ to obtain

$$
\tau \approx 14.14 d_{0}-7.17
$$

which is an extremely simple closed-form expression to estimate the required truncation numbers for small boxes and low-frequency problems. Note that (19) will only yield values larger than unity for $d_{0}>0.58$ or $\epsilon_{d}<0.26$ due to its affine nature, which should be useful for almost every scenario.

To illustrate the accuracy of the closed-form expressions and EBF given in (3), we analyzed the average relative 


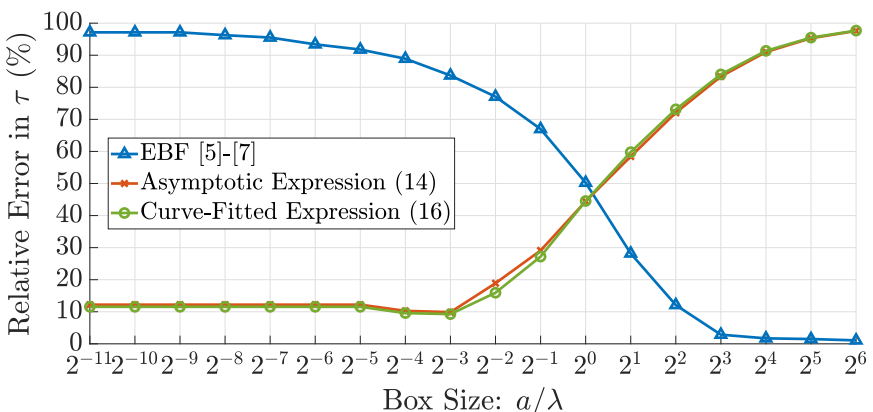

Fig. 2. Relative error of truncation numbers obtained via EBF [5]-[7], (14), and (16) for different box sizes and translation distances.

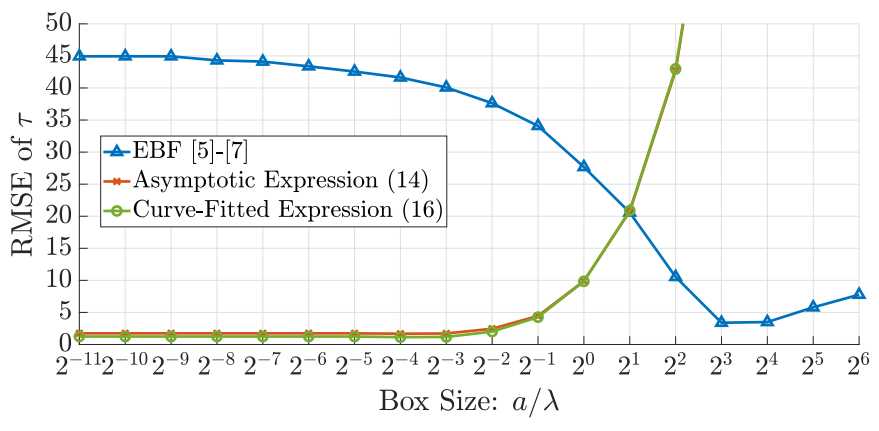

Fig. 3. RMSE of truncation numbers obtained via EBF [5]-[7], (14), and (16) for different box sizes and translation distances.



Fig. 4. Relative error of truncation numbers obtained via EBF [5]-[7], (15), and (19) for different box sizes for a one-box-buffer scheme.

error and root-mean-square error (RMSE) of the truncation numbers over the same $\epsilon_{d}, \vec{w}$, and $\vec{v}$ values that we have listed previously, with an even greater range of box sizes that include electrically large boxes. The errors for the truncation numbers are shown in Figs. 2 and 3 for the variable-translation case with (14) and (16) and in Figs. 4 and 5 for the one-box-buffer scheme using (15) and (19). For all cases, RMSE values for $\mathrm{EBF}$ are calculated for only $w=2 a$, as EBF is not able to handle different translation distances within the same parent box of MLFMA.

As shown in Figs. 2-5, EBF achieves relatively low errors for large boxes, and the proposed closed-form expressions (14), (15), and (16)-(19) perform well for small boxes. Note that, although the proposed expressions for low frequencies exhibit a slightly large relative error, they perform much better in terms of RMSE. This is due to truncation numbers being much smaller at low frequencies compared to high frequencies.

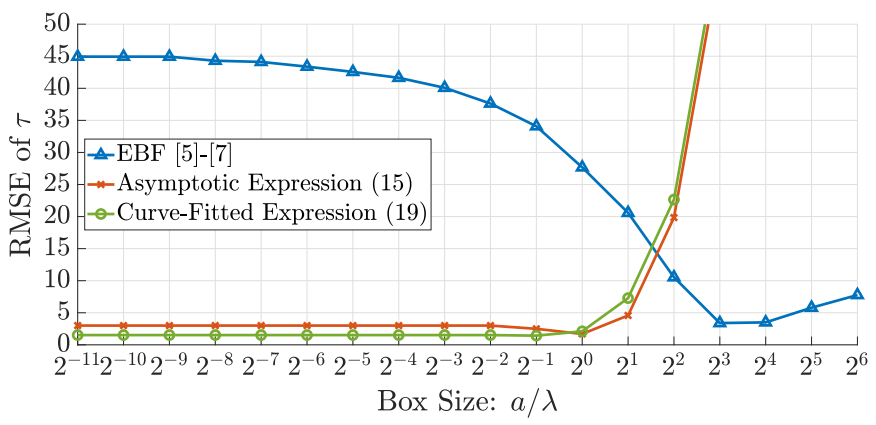

Fig. 5. RMSE of truncation numbers obtained via EBF [5]-[7], (15), and (19) for different box sizes for a one-box-buffer scheme.

For example, even an RMSE of 1.2 for $a=\lambda / 2048$ has led to a relative error of $11 \%$ in Figs. 2 and 3. The converse is true for $\mathrm{EBF}$ at high frequencies where the truncation numbers are very large, leading to relatively high RMSE values although their relative errors are very low. Another important observation from Figs. $2-5$ is that the curve-fit expressions outperform the asymptotic expressions, especially for the one-box-buffer scheme shown in Figs. 4 and 5, as they are based on the accurate error control expression in (4). As the truncation numbers are selected for the one-box-buffer scheme in practice, Figs. 4 and 5 show the power of the extremely simple expression that we have provided in (19).

As shown in Figs. 4 and 5, it is best to use the proposed closed-form expressions for relatively small box sizes, i.e., $a<2.8 \lambda$, whereas EBF should be used exclusively for $a>2.8 \lambda$, where $a=2.8 \lambda$ is approximately the point where the errors of EBF and small-box expressions coincide. Also, the errors are relatively larger in the region $a \in[\lambda, 4 \lambda]$ for the combined usage of small-box expressions and EBF. Therefore, practitioners of MLFMA should be aware that these expressions are relatively erroneous in this particular region. We would like to emphasize again that the error control formulation of (4) is valid for all box sizes and should be used if a multiple-precision framework could be implemented by the practitioners. Since a multiple-precision framework may not be available for most cases, we provide detailed design curves that can be used to estimate the achievable errors of MLFMA for commonly available fixed-precision computers in the next section.

\section{ERROR ANALYSIS OF MLFMA}

The simplified error control scheme with closed-form expressions (16)-(19) together with the machine precision estimation method given in [4] can be used to estimate the minimum achievable errors (denoted as $\epsilon_{\min }$ ) or minimum translation distances for a given fixed machine precision. Note that, in this section, the machine precision levels are provided as decimal digits of precision in a floating-point standard [13], where single, double, and quadruple precisions correspond to approximately 7,16 , and 34 decimal digits of precision, respectively.

Using (16)-(19) for subwavelength box sizes $(a<\lambda)$ and (4) for all other cases and desired errors larger than 


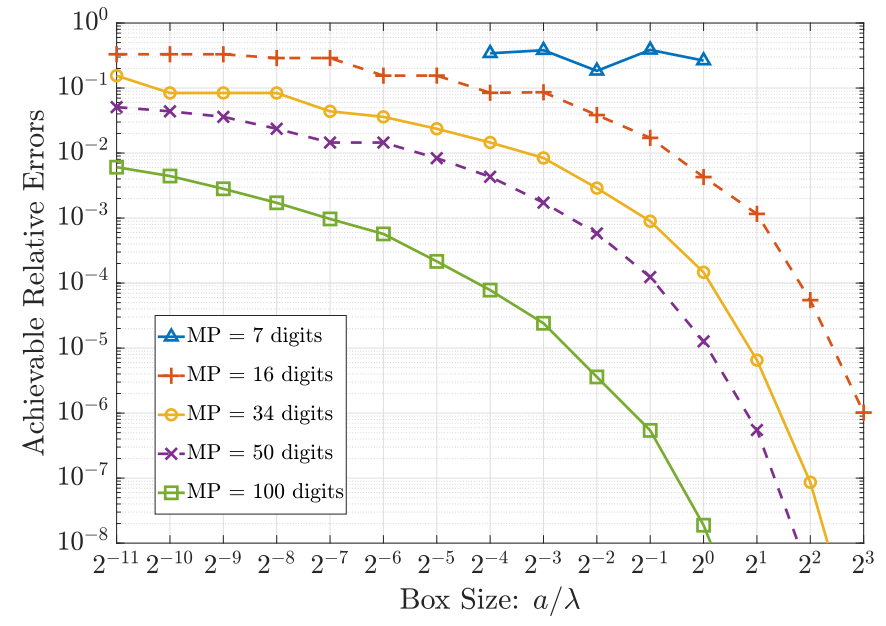

Fig. 6. Minimum achievable errors versus the box size in a one-box-buffer scheme (i.e., $w=|\vec{w}|=2 a$ ).

$\epsilon_{d}>0.26$, we present three design curves to provide a helpful guideline for MLFMA practitioners. Note that, although EBF is valid for large boxes, it slightly overestimates the truncation numbers due to simplification of the Legendre polynomials' exact value as unity [5], while (16)-(19) do not suffer from such a problem. Therefore, to provide the most accurate design curves possible, we have used (4) for box sizes greater than one wavelength.

First, we investigate the minimum achievable error versus the box size for various machine precision levels in a onebox-buffer scheme (i.e., $w=2 a$ ). Fig. 6 shows the achievable error bounds of MLFMA on fixed-precision computing platforms for a wide range of box sizes. For scattering and radiation problems, where only far-zone radiation characteristics are of interest, relatively large errors in Green's function can be tolerated. For example, assuming an error bound of $\epsilon=0.33$, both single- and double-precision platforms are able to handle subwavelength box sizes (a minimum of $2^{-4} \lambda$ and $2^{-11} \lambda$, respectively). On the other hand, for problems where accurate current distributions and network parameters are required (e.g., antenna problems), relatively low error bounds for Green's function must be enforced. For example, if an error bound of $\epsilon=0.01$ is selected, both single and double precisions fail to handle subwavelength box sizes, as shown in Fig. 6. As a result, higher precision levels may be required to solve antenna problems with large numbers of MLFMA levels that include subwavelength translation distances.

An important observation to note in Fig. 6 is that, for the single-precision case ( 7 decimal digits), the minimum achievable errors are only reported for $a \in\left[2^{-4} \lambda, \lambda\right]$, meaning that the error is not bounded outside this region. Specifically, single-precision is not enough to handle the spherical Hankel functions, leading to low-frequency breakdown for box sizes smaller than $2^{-4} \lambda$. On the other hand, for box sizes larger than $\lambda$, relatively small numerical integration weights cannot be handled by single precision as the number of spherical harmonics increases. Nonetheless, single precision

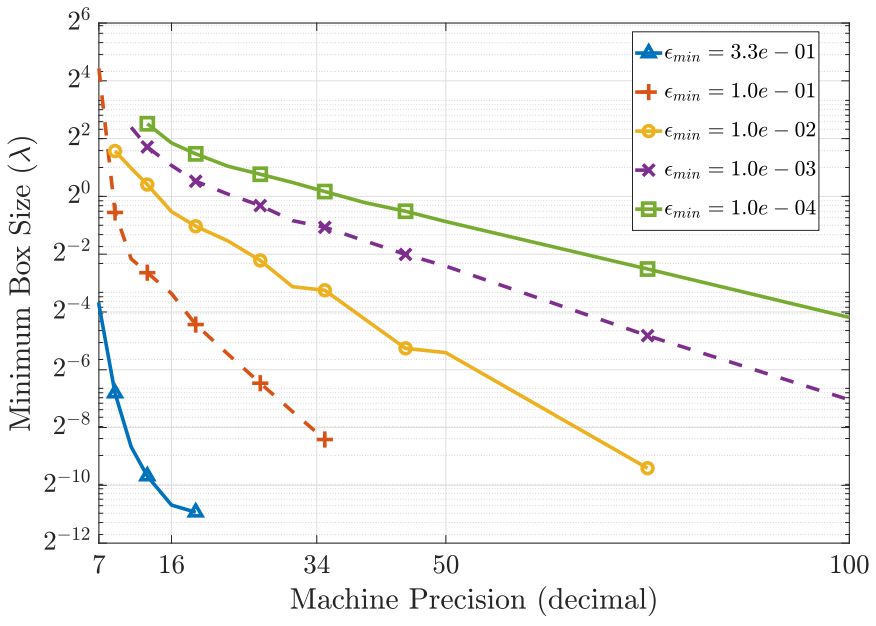

Fig. 7. Minimum box size versus the machine precision to achieve different desired error thresholds for $w=|\vec{w}|=2 a$, i.e., the one-box-buffer scheme

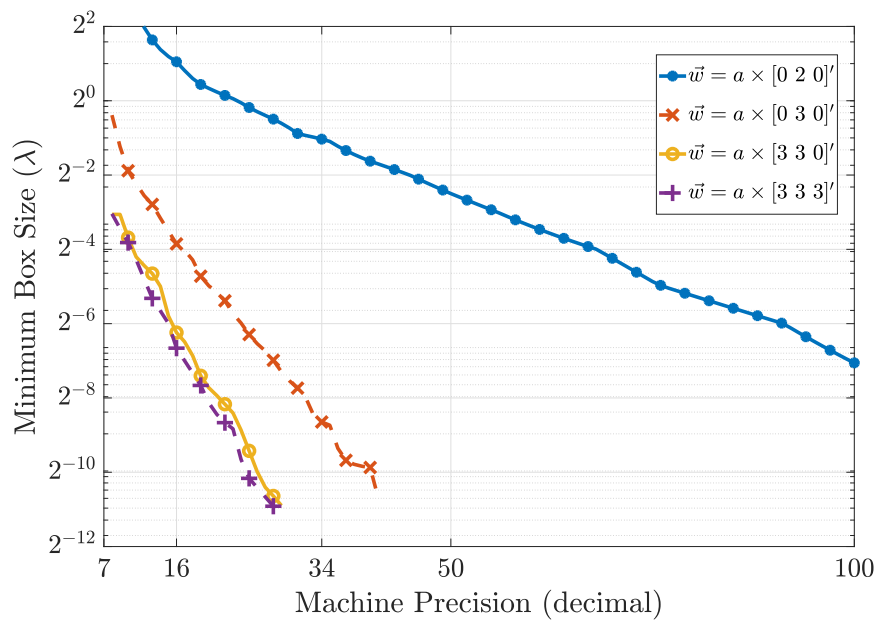

Fig. 8. Minimum box size versus the machine precision for different translation vectors to achieve a desired error of $\epsilon_{\min }=10^{-3}$.

can possibly handle larger boxes (and achieve lower errors) if special care is taken to prevent overflow/underflow during the computation of the translation operator, as explained in Section II.

As shown in Fig. 7, our second design curve illustrates the minimum box size that can be used in MLFMA given the desired relative error threshold. In addition to demonstrating new information on the achievable error levels in MLFMA, the results in Fig. 7 further verify the conclusions drawn from Fig. 6, i.e., single and double precisions fail to accurately handle subwavelength boxes for relatively low error levels, and they should only be considered for larger box sizes or problems where large errors of Green's function can be tolerated. Also, note that the graphs only show the box sizes included in the analysis (i.e., $a \in\left[2^{-11} \lambda, 2^{4} \lambda\right]$ ), and the minimum box sizes are expected to decrease further as the machine precision increases.

In our third and final design curve, we illustrate the minimum box size that can be handled for a given translation 
distance in Fig. 8. Different translation distances are selected among possible far-zone translations inside a parent box of an MLFMA tree structure. As expected, Fig. 8 shows that the achievable minimum box size decreases as the magnitude of the translation vector gets larger since the overflow problem due to the spherical Hankel function in the translation operator becomes less severe as $w$ increases [4]. Thus, lower precision levels can be used for large translation distances even if higher precision levels may be required for the one-box-buffer scheme. Also, similar to Fig. 7, the minimum box sizes are expected to decrease when higher machine precision levels are used though they are limited to the analyzed box sizes in Fig. 8.

\section{CONCLUSION}

We have proposed and validated simplified closed-form expressions for the accurate error control of MLFMA that is valid for low-frequency problems. Together with the commonly used EBF, our proposed expressions can be implemented easily for broadband error analysis of MLFMA although our previously reported error control scheme that requires a multiple-precision framework should be considered as the gold standard. Moreover, we presented a thorough investigation of achievable error limits in MLFMA while taking into account the machine precision used in the computing platform. We believe that the practitioners of MLFMA solvers can use the proposed expressions and presented design curves in this article as a helpful guideline to estimate the error limits of their implementation on any platform, whether they use fixedor variable-precision arithmetic computing platforms.

\section{REFERENCES}

[1] R. Coifman, V. Rokhlin, and S. Wandzura, "The fast multipole method for the wave equation: A pedestrian prescription," IEEE Antennas Propag. Mag., vol. 35, no. 3, pp. 7-12, Jun. 1993.

[2] J. Song, C.-C. Lu, and W. Cho Chew, "Multilevel fast multipole algorithm for electromagnetic scattering by large complex objects," IEEE Trans. Antennas Propag., vol. 45, no. 10, pp. 1488-1493, Oct. 1997.

[3] L. Greengard, J. Huang, V. Rokhlin, and S. Wandzura, "Accelerating fast multipole methods for the Helmholtz equation at low frequencies," IEEE Comput. Sci. Eng., vol. 5, no. 3, pp. 32-38, 1998.

[4] M. Kalfa, O. Ergul, and V. B. Erturk, "Error control of multipleprecision MLFMA," IEEE Trans. Antennas Propag., vol. 66, no. 10, pp. 5651-5656, Oct. 2018.

[5] S. Koc, J. Song, and W. C. Chew, "Error analysis for the numerical evaluation of the diagonal forms of the scalar spherical addition theorem," SIAM J. Numer. Anal., vol. 36, no. 3, pp. 906-921, Jan. 1999.

[6] J. Song and W. Cho Chew, "Error analysis for the truncation of multipole expansion of vector Green's functions [EM scattering]," IEEE Microw. Wireless Compon. Lett., vol. 11, no. 7, pp. 311-313, Jul. 2001.

[7] W. C. Chew, J.-M. Jin, E. Michielssen, and J. M. Song, Fast and Efficient Algorithms in Computational Electromagnetics. Boston, MA, USA: Artech House, 2001.

[8] M. L. Hastriter, S. Ohnuki, and W. C. Chew, "Error control of the translation operator in 3D MLFMA," Microw. Opt. Technol. Lett., vol. 37, no. 3, pp. 184-188, May 2003.

[9] L. Wu et al., "MLACE-MLFMA combined with reduced basis method for efficient wideband electromagnetic scattering from metallic targets," IEEE Trans. Antennas Propag., vol. 67, no. 7, pp. 4738-4747, Jul. 2019.

[10] Multiprecision Computing Toolbox for MATLAB. Accessed: Aug. 11, 2017. [Online]. Available: http://www.advanpix.com

[11] M. Abramowitz and I. A. Stegun, Handbook of Mathematical Functions With Formulas, Graphs, and Mathematical Tables. New York, NY, USA: Dover, 1964.

[12] O. Ergul and L. Gurel, "Efficient solutions of metamaterial problems using a low-frequency multilevel fast multipole algorithm," Prog. Electromagn. Res., vol. 108, pp. 81-99, 2010.

[13] IEEE Standard for Floating-Point Arithmetic, Standard 754-2019, 2019.

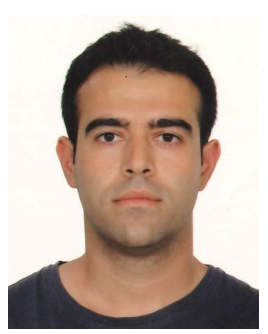

Mert Kalfa (Member, IEEE) received the B.Sc., M.Sc., and Ph.D. degrees from the Department of Electrical and Electronics Engineering, Bilkent University, Ankara, Turkey, in 2010, 2013, and 2020, respectively.

$\mathrm{He}$ is currently a Principal Research Engineer with the Huawei Turkey Research and Development Center, İstanbul, Turkey, and a Post-Doctoral Visiting Research Associate with Bilkent University. His research interests include, but not limited to, computational electromagnetics, phased array antennas, compressive sensing, and semantic communications.



arrays

Dr. Ertürk was the Secretary/Treasurer of the IEEE Turkey Section and the Turkey Chapter of the IEEE Antennas and Propagation, Microwave Theory and Techniques, Electron Devices, and Electromagnetic Compatibility Societies. He was a recipient of the 2005 URSI Young Scientist, the 2007 Turkish Academy of Sciences Distinguished Young Scientist, and the 2020 Bilkent University Distinguished Teaching awards.

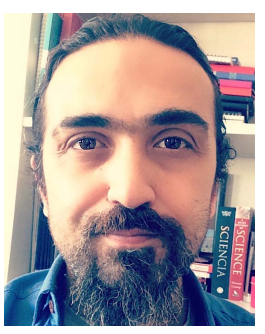

Özgür Ergül (Senior Member, IEEE) received the B.Sc., M.S., and Ph.D. degrees from Bilkent University, Ankara, Turkey, in 2001, 2003, and 2009 , respectively, all in electrical and electronics engineering.

He is currently a Professor with the Department of Electrical and Electronics Engineering, Middle East Technical University (METU), Ankara. His research interests include the development and implementation of fast and efficient solvers for electromagnetic problems. 